\title{
COMMON-OFFSET DEPTH MIGRATION AS A VELOCITY ANALYSIS TOOL
}

\author{
Shein S. Wang, Robert T. Baumel, Douglas W. Hanson, \\ David W. Bell, Mark Boyd, Thomas D. Cavanaugh, V. Dale Cox \\ Peter S. D'Onfro, Javaid A. Durrani and Larry A. Standlee \\ Conoco Inc. \\ PO Box 1267 \\ Ponca City OK 74603 \\ USA
}

\begin{abstract}
We imaged the Marmousi dataset using an efficient Kirchhoff prestack depth migration algorithm combined with a layer-stripping velocity analysis technique. The method relies on repeated common-offset test migrations to build a velocity model systematically from the top down. Results of these common-offset depth migrations are sorted into common image-point displays. By observing residual moveout as a function of offset in these displays, we determine velocities and boundary locations for each layer. The assumed model is 'blocky' with constant layer velocities. Application of this method to the Marmousi data produced a good quality image except in the central region with its large-velocity-contrast fault blocks. Our data treatment for the EAEG workshop consisted of wavelet processing, followed by our layer-stripping depth migration/model building, and residual processing. Some additional improvement has been obtained by reprocessing after the workshop. Although a blocky model with constant-velocity layers is a highly idealized model, our success in applying it to this complex synthetic dataset makes us more comfortable in using this method to image datasets from the real world.
\end{abstract}

\section{INTRODUCTION}

Our procedure for building velocity models for depth migration uses common-offset depth migration combined with a top-down layer-stripping velocity analysis technique. The technique utilizes a stable and fast (12 minutes CPU time for the Marmousi prestack dataset on a CRAY X-MP) Kirchhoff depth migration algorithm, which allows many trial velocities to be tested.

We processed the Marmousi dataset in three main steps: (1) pre-processing to remove the seismic wavelet, (2) iterative depth migration and layer-stripping velocity analysis, and (3) postmigration processing including a residual moveout correction. The second step, namely, iterative construction of the migration velocity model, comprised the bulk of the effort, and will also receive most of the discussion in this report.

Our velocity-depth models consist of constantvelocity layers with arbitrarily dipping boundaries. We construct such a model systematically, one layer at a time, from the top down. With the model determined to any given layer, common-offset test migrations are run for various (constant) velocities below that layer. The common-offset results are sorted into common image-point gathers, which help determine the velocity and location of the next layer.

This velocity analysis is quite sensitive. It is usable whenever the image-point gathers are sufficiently coherent to display event moveout over an adequate range of offsets. However, poor data quality or structural complexity can cause the method to break down at sufficiently great depths.

For the Marmousi dataset, near the east and west ends of the line, our systematic layer-stripping method was usable (and yielded accurate veloci- 


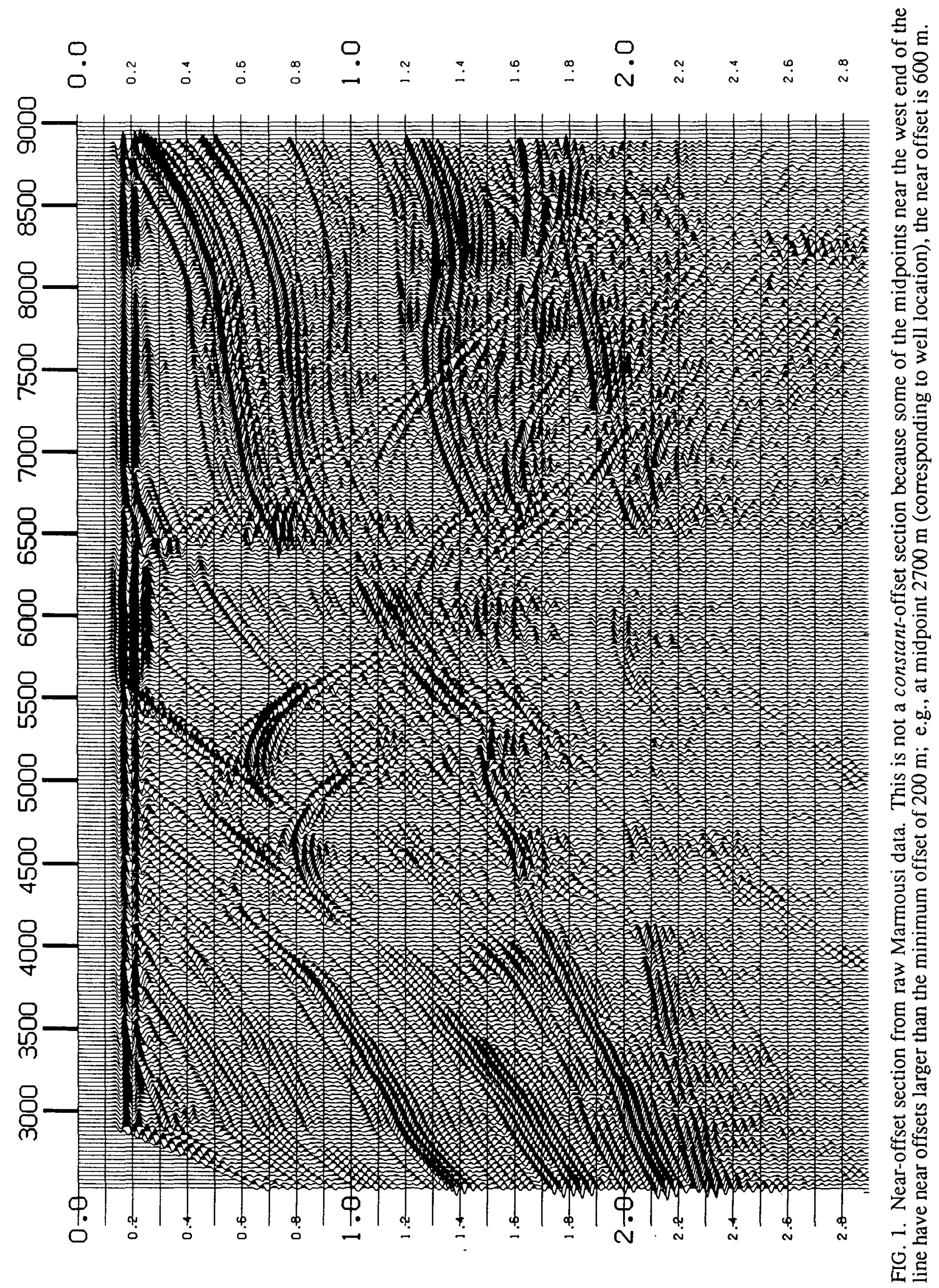


ties) down to the top of salt at depths greater than $2000 \mathrm{~m}$. In the faulted central portion, however, we could apply the method (at least during the limited time before the Workshop) down to only about $1200 \mathrm{~m}$. Below these depths, we used a more 'brute force' approach of migrating the region with a suite of velocities and picking the velocity that gave the clearest image.

We believe that this 'brute force' method is similar in accuracy to the 'focusing analysis' method (Faye and Jeannot, 1986), while our more systematic approach based on common image-point gathers allows much greater accuracy and reliability by presenting the components of the stacked depth image as a function of offset for examination.

The present report will describe our treatment of the Marmousi dataset as part of the blind experiment prior to the Copenhagen workshop. We will also describe some 'semi-blind' work conducted after the workshop to see how much better we might have done if we had looked at the data a little more carefully.

\section{DATA PROCESSING}

\section{Wavelet Processing}

A first attempt to deconvolve the data using a wavelet derived from the VSP was disappointing. We estimated the wavelet by picking the VSP first arrivals, and stacking them. But while traces deconvolved with this wavelet did appear to be somewhat improved close to the well that the VSP came from, traces far from this well actually acquired a more ringy appearance than the raw data.

We think this happened because the 'wavelet' in the VSP first arrivals included effects such as water-bottom reverberation, which would vary from place to place. The effect of the water layer could presumably be removed by modeling, but we elected to try the following simpler approach:

This approach consisted of (1) deterministic removal of the marine source signature which is common to every trace in the dataset, followed by (2) standard statistical ('spiking') deconvolution to remove the remaining 'wavelet' due to ghosting, reverberation, etc., which we expect to be, at least approximately, minimum phase.

To back out the source signature, we generated synthetic traces from the velocity and density logs furnished for the well at location $2700 \mathrm{~m}$. Figure 1 shows a near-offset section of the raw Marmousi seismic data. The $2700 \mathrm{~m}$ well location occurs near the extreme west end of the seismic line, where the data are not at full fold. Specifically, at midpoint $2700 \mathrm{~m}$, the smallest offset is $600 \mathrm{~m}$.

Figure 2 is a synthetic gather generated by plane-layered modeling from the $2700 \mathrm{~m}$ well logs. This synthetic includes source and receiver arrays, source and receiver ghosting, and all orders of multiples; thus, we think it contains all the effects in the Marmousi traces except for the source signature and geometric structure (i.e., dipping layers).

Figure 3 illustrates a transfer function analysis of the $600 \mathrm{~m}$ offset trace from Figure 2 against the matching trace at midpoint $2700 \mathrm{~m}$ from Figure 1. This transfer function analysis yields the estimated source signature shown in Figure 3c. The true Marmousi signature is also shown for comparison in Figure 3d.

Step (1) of our deconvolution procedure was to remove the estimated source signature shown in Figure 3c. The effect of this operation on the nearoffset traces is shown in Figure 4a. Step (2) was a standard spiking deconvolution. The final result is illustrated in Figure 4b.

Figure $4 c$ is a time migration of the deconvolved near-offset section of Figure $4 \mathrm{~b}$. This is a fairly crude time migration; the velocity used for it was not derived by velocity analysis, but by simple linear interpolation between well velocities at the ends of the line. Nevertheless, it does correctly indicate the nature of the shallow faulting, and was therefore helpful as we began building our depth migration velocity model.

\section{Kirchhoff Migration Algorithm}

Kirchhoff depth migration works by 'broadcasting' each event in a trace to every possible subsurface reflection point consistent with the source and receiver locations and the travel time of the event. As an example, Figure 5 shows the result of broadcasting only two of the Marmousi traces in 


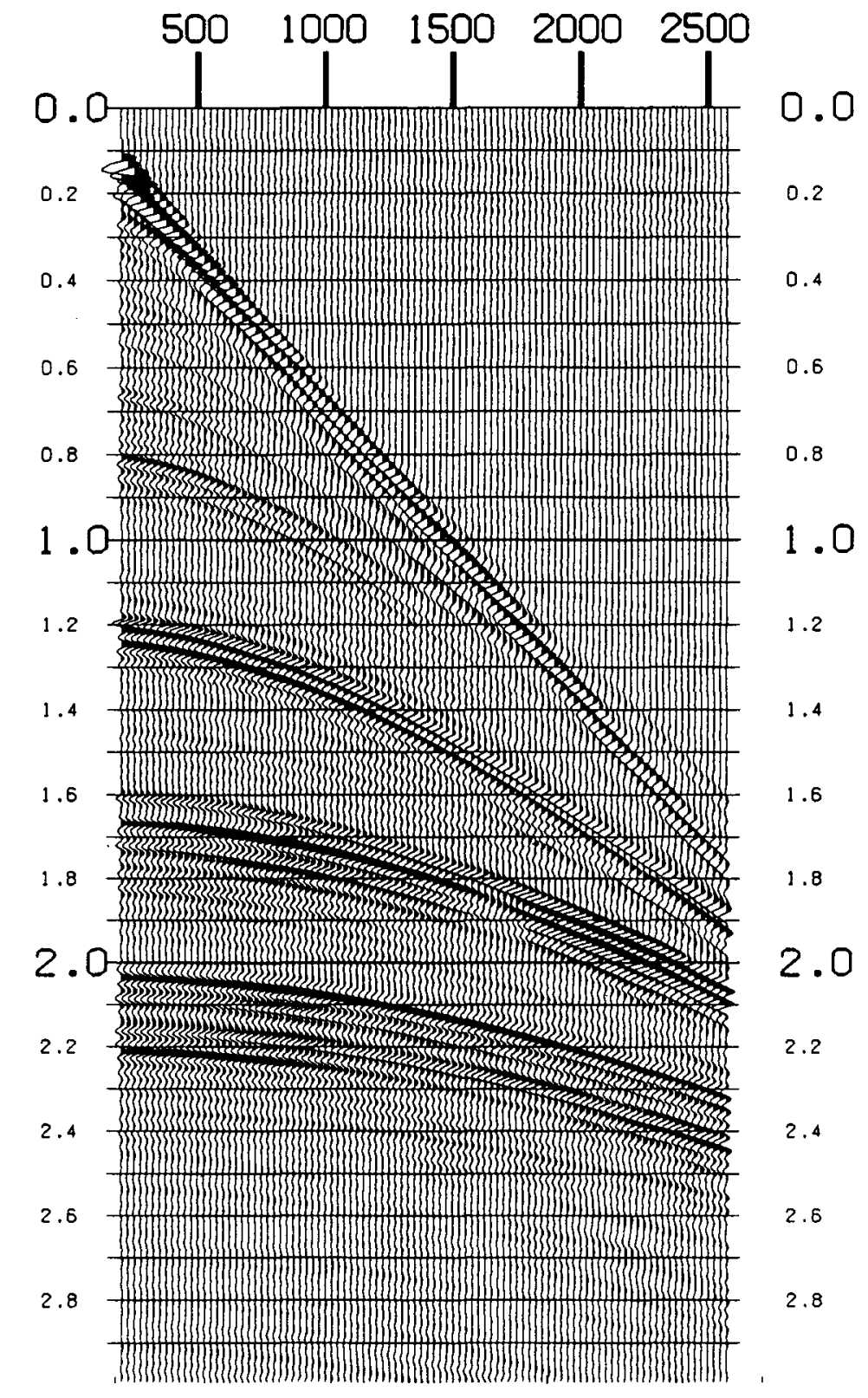

FIG. 2. Plane-layered synthetic from well 2700. This synthetic incorporates source and receiver arrays, source and receiver ghosting, and all orders of multiples. The wavelet is a band-limited spike. 


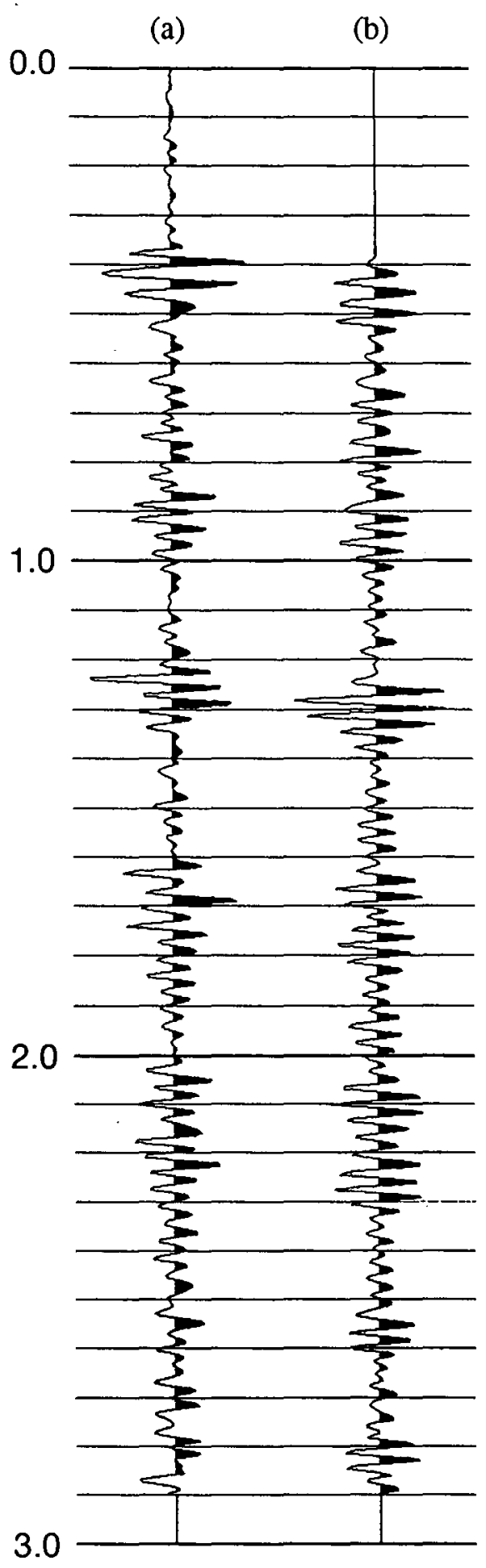

(c)

(d)

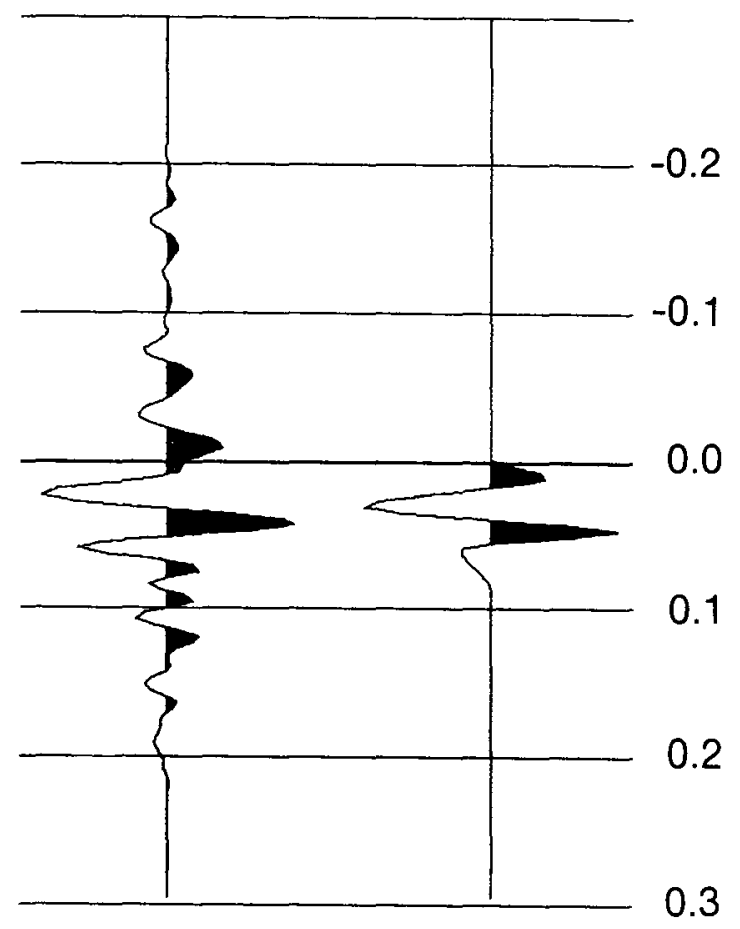

FIG. 3. Transfer function analysis to estimate source signature. Transfer function is computed between (a) the plane-synthetic trace from Figure 2 at offset $600 \mathrm{~m}$, and (b) the Marmousi trace at midpoint $2700 \mathrm{~m}$ and offset $600 \mathrm{~m}$, yielding (c) the estimated signature. (d) is the true Marmousi source signature shown for comparison. 


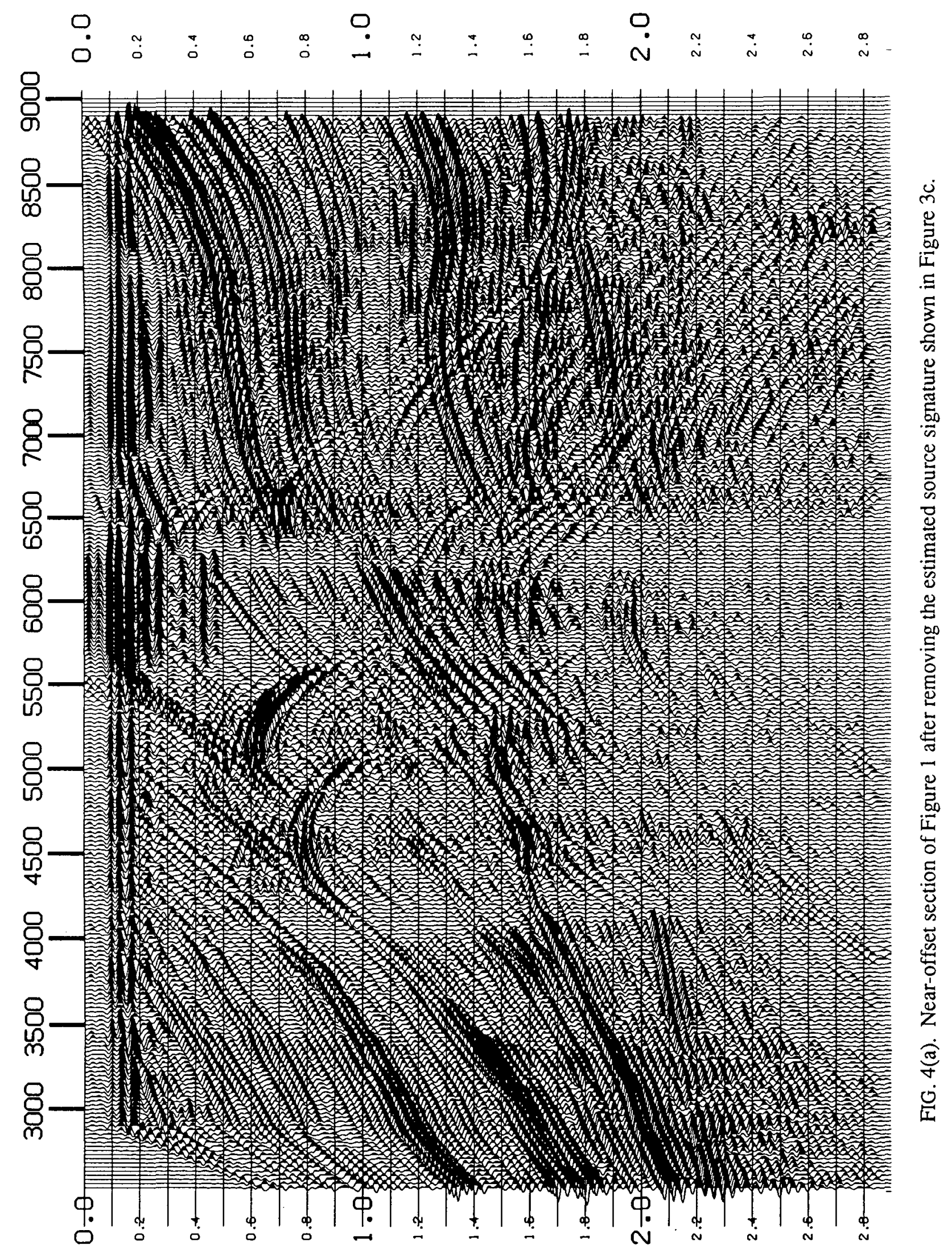




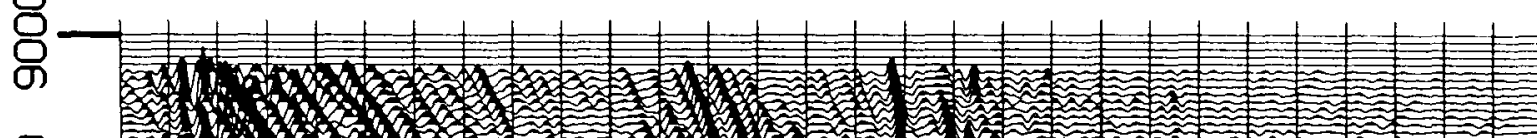

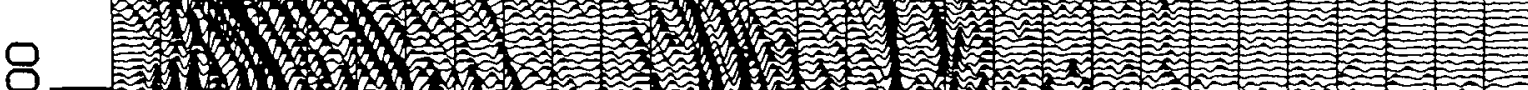
ஸि- ${ }_{\infty}$

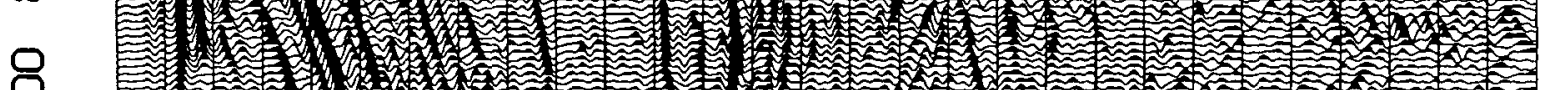
๑一, ○一 Q ○一

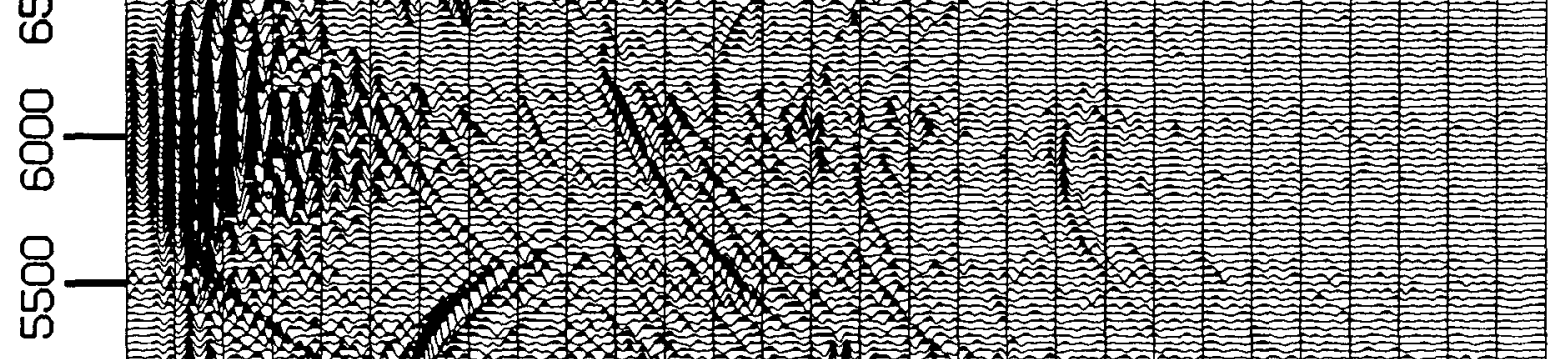
Q ๑

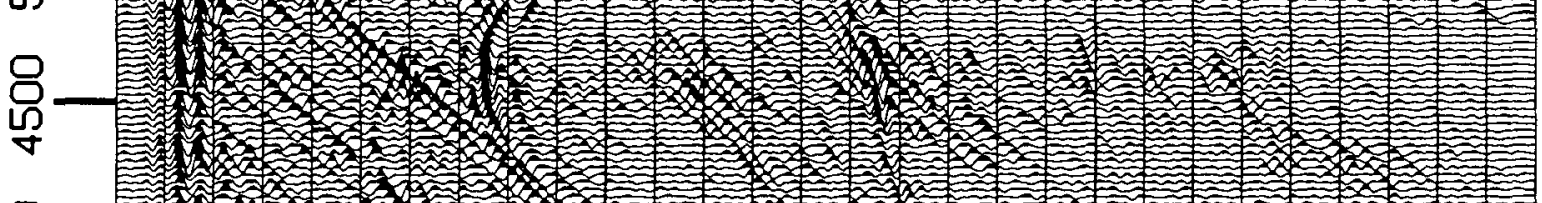

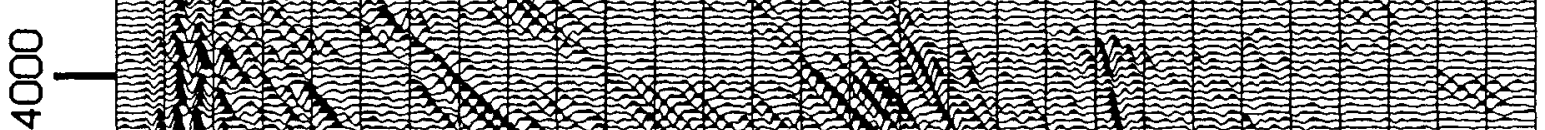
O. 9.

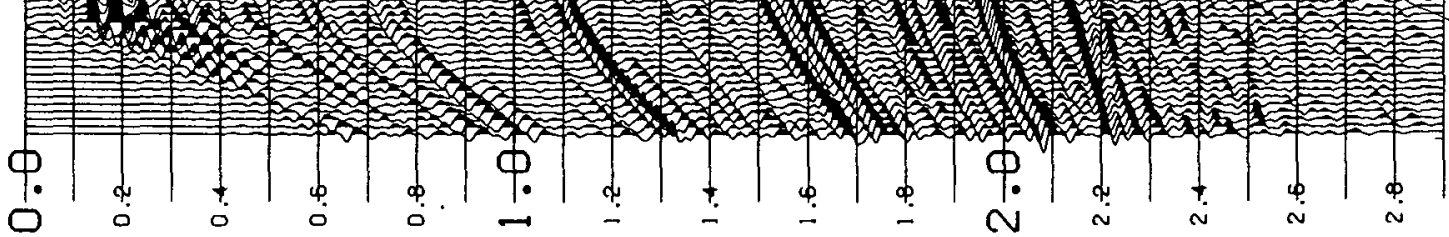




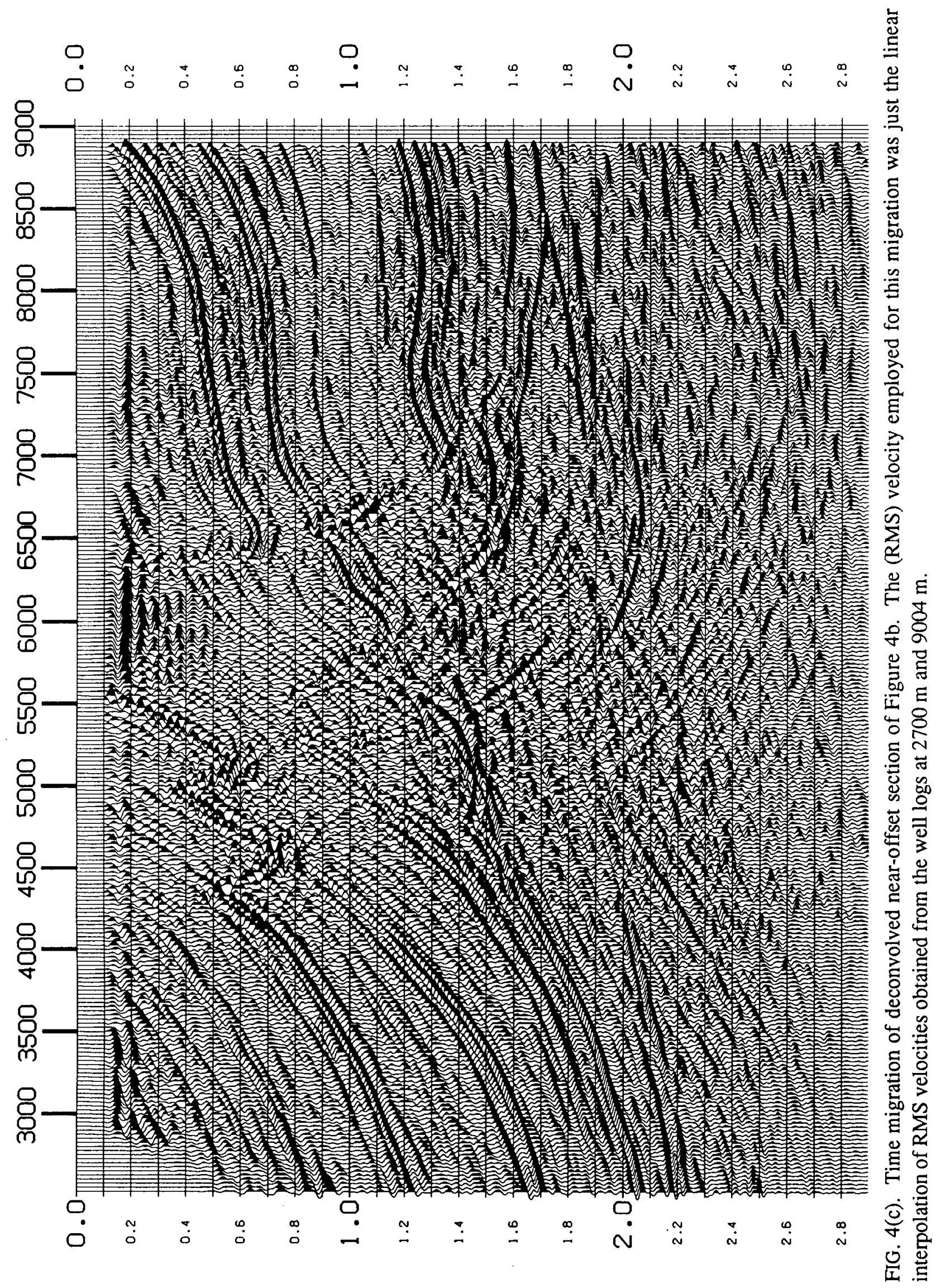


this fashion. When sufficiently many traces have been migrated, constructive and destructive interference build up an image of the subsurface reflectors.

In order to broadcast the traces, travel times must be computed between surface points and each subsurface point. Our algorithm generates and stores a separate travel time table for each source or receiver location. Later, for other traces with the same receiver location, the table is re-used, and these traces are migrated quickly.

In this way, the time spent generating the travel time table becomes a small portion of the total cost, allowing us to use accurate methods of travel time generation such as finite difference solution of the eikonal equation (Van Trier and Symes, 1990) or graph theory (Moser, 1989). Our program can depth migrate about 20-40 traces per second on the CRAY X-MP 14, making it possible to use a large number of trial velocities in the test migrations.

\section{Common Offset Depth Migration}

When seismic data are sorted into common offset panels, each offset can be processed as if it were an individual seismic section. Common offset depth migration converts each offset panel into a depth section, each of which represents the same subsurface structure. With a perfect velocity model, all the offset sections would yield identical images. For real seismic data, it is normally impossible to obtain a perfect velocity model, and the migrated offset sections are then different.

When these common offset depth sections are sorted into common image-point gathers, differences in the images with offset can be readily seen. A flat imaged event indicates that the reflector was migrated to the same depth on each offset panel; hence, the velocity model used in the migration is correct. If the imaged event appears too deep at far offsets, the migration velocity was too fast. If the imaged event appears too shallow at far offsets, the velocity was too slow.

By retaining image-point gathers after common offset depth migration, we can study the residual moveouts. These provide information for refining the velocity model, and also for improving the depth image without re-migrating.

\section{Layer Stripping Velocity Analysis}

When a velocity-depth model has been determined down to a given interface, test migrations are performed using a suite of constant velocities below this interface (holding the model fixed above the interface). A reflector below this interface is chosen to serve as the next deeper layer boundary. The correct velocity of the layer between these two horizons is the velocity at which the reflector defining its lower boundary has least moveout in the image-point gathers. The correct location of this lower boundary is the position of the reflector's image when migrated with the correct velocity.

Figures $6 \mathrm{a}, 6 \mathrm{~b}$, and $6 \mathrm{c}$ illustrate this procedure on a portion of the Marmousi data. The left half of each figure shows the migrated image (stack of migrated common-offset panels) for $x$ range $2500-4500 \mathrm{~m}$, while the right half shows the imagepoint gather at $x=3250 \mathrm{~m}$. There are 64 offsets in the gather and the near offset is to the left. The model has been determined down to the interface defined by reflector I, while reflector II has been chosen to define the next deeper boundary. We must determine the velocity between interfaces I and II, and the correct location of interface II.

The three figures $(6 a, 6 b$, and $6 c)$ were generated by migrating with different velocities below interface $I$. Figure $6 \mathrm{~b}$ was migrated with the velocity we chose as correct, as indicated by the near-flatness of reflector II in the image-point gather in figure $6 \mathrm{~b}$. In Figure $6 \mathrm{a}$ the velocity is decreased by $5 \%$. The image is shallower at far offsets, indicating that this velocity is too slow. In Figure $6 \mathrm{c}$ the velocity is increased by $5 \%$. The image is deeper at far offsets, indicating that this velocity is too fast.

Given that figure $6 \mathrm{~b}$ provides the correct velocity between interfaces I and II, the correct location of interface II is picked from the migrated image on the left side of figure $6 \mathrm{~b}$.

\section{Completing the Velocity Model}

As mentioned earlier, our systematic layer- 


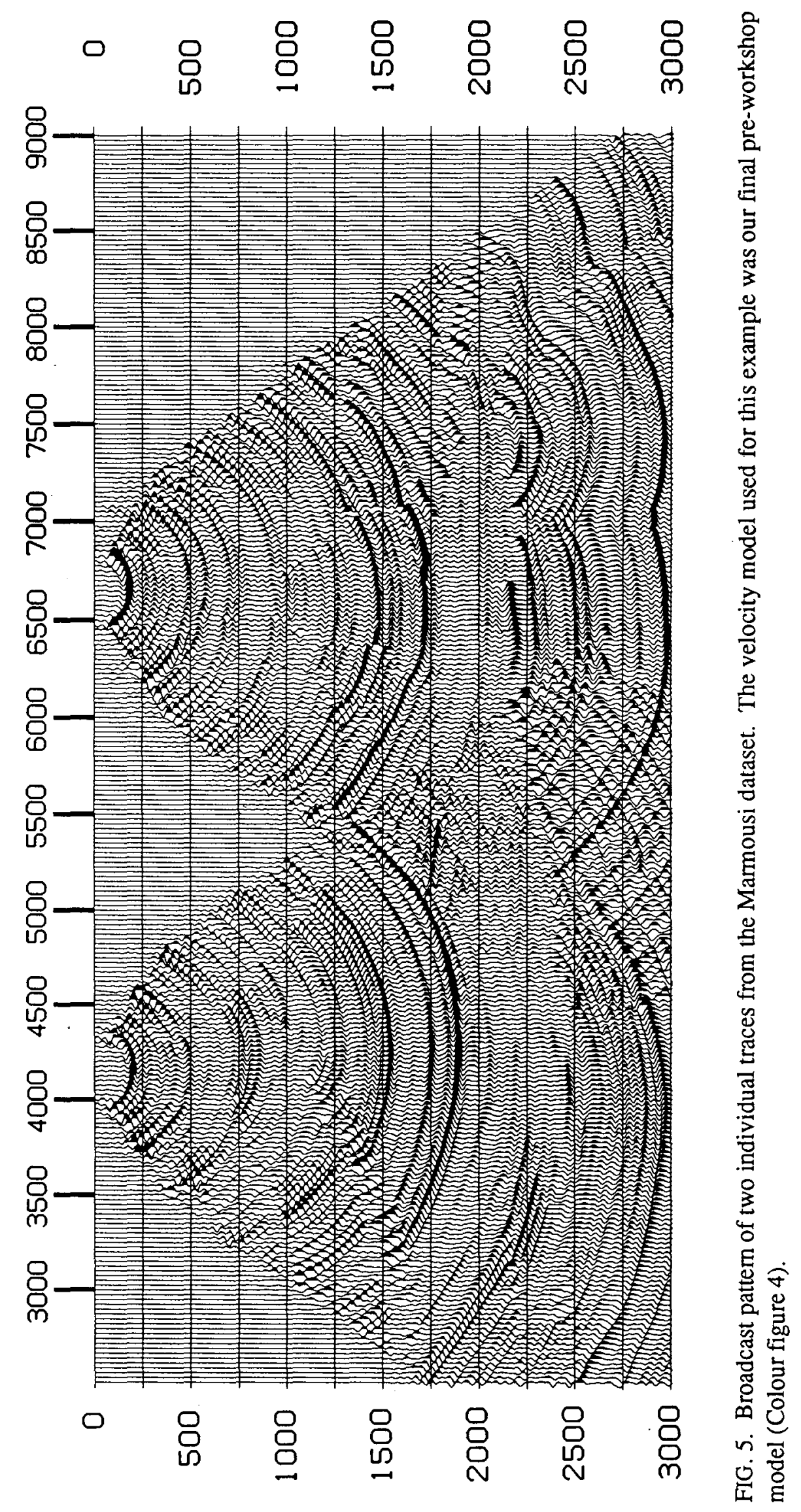




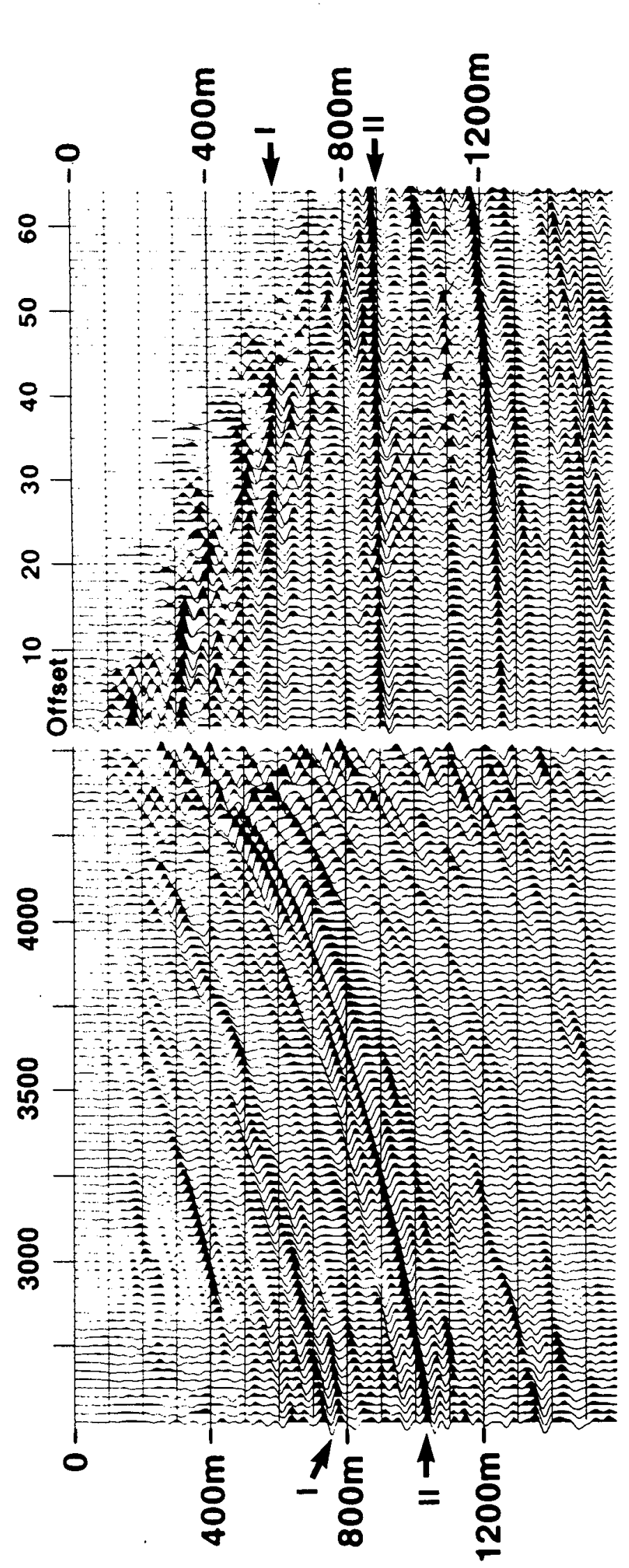

:

文 吉

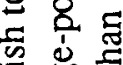

3 年

छ

들 올

등

농 엉

형 형

昰言

음

$\doteq 5$

를

헝옹 엉

茎量

홍.

员:

氙

$=0$

.

믕ㅇㅁ

륭 엉

艺可

可

윰

牢

总

on:

.

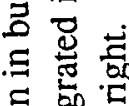

. 을.

.․ㅗㅇ

븡

㟧

范《

気

बे

용

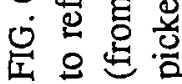




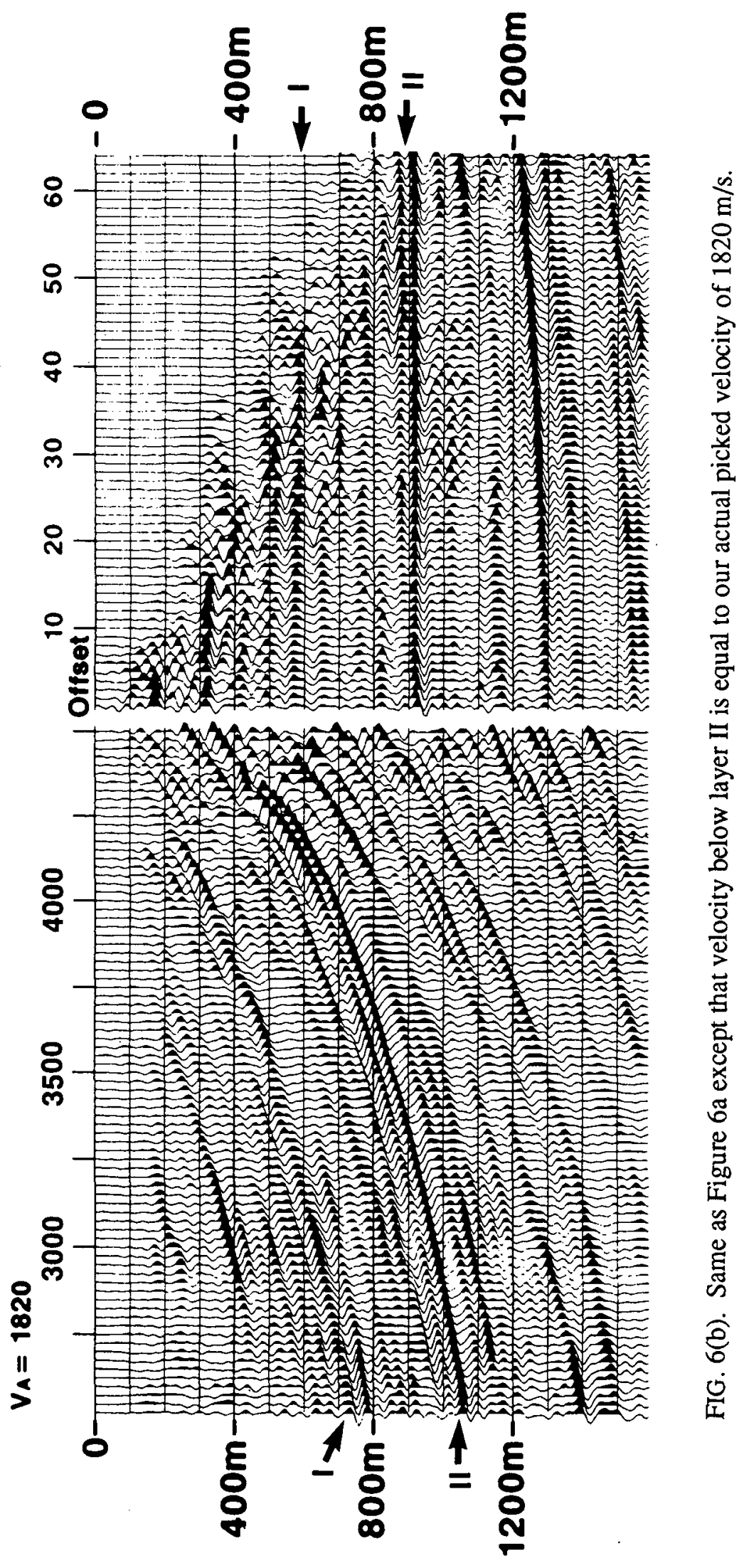




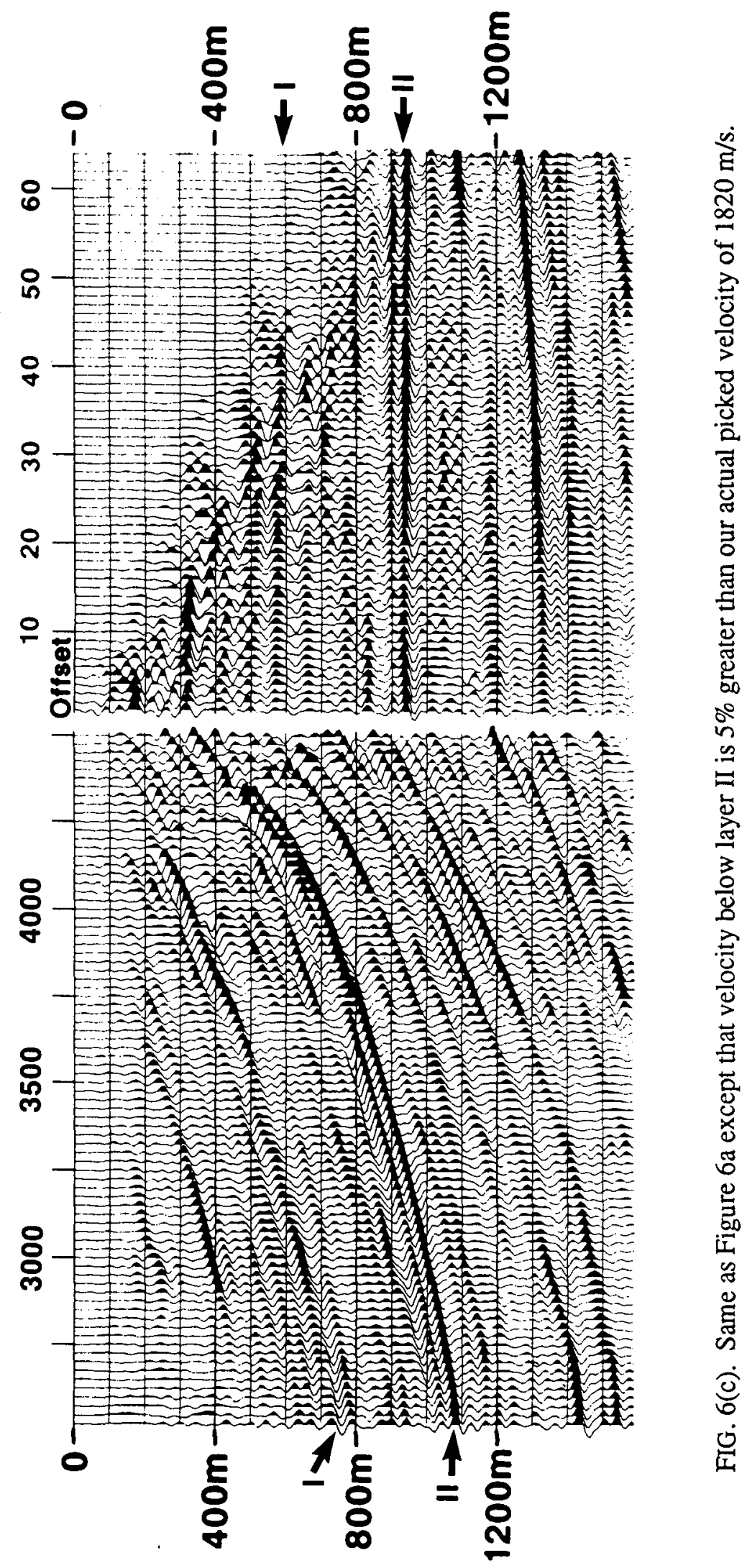


stripping procedure can break down at sufficiently great depths, depending on structural complexity. This occurred at about depth $1200 \mathrm{~m}$ in the faulted central region of the Marmousi model. To extend the model deeper, we used the 'brute force' method of simply trying different velocities to see which produced the clearest image.

This approach can be very non-unique, and will therefore yield good results only when constrained by good geological knowledge. We made two key geological assumptions at this point:

(1) Based on our reading of the "Geological model definition" supplied with the Marmousi dataset, we assumed that the unconformity above the Phase I carbonates is basically flat, even if slightly dipping.

(2) We expected that a domed salt structure must exist in the central portion of the model to explain the shallower growth faulting.

As we know now, the first assumption was correct, while the second was wrong. (Note: We did know, as a result of phoning IFP, that all 'salt' used in this exercise had the unrealistically high velocity of $5500 \mathrm{~m} / \mathrm{s}$. Consequently, the 'salt' we tried putting in the central part of our model had this very high velocity.)

Colour figure 4 shows our final velocity model as presented at the Workshop, while figure 7 shows the migrated image obtained with that model. The central part of our model in Colour figure 4 includes $5500 \mathrm{~m} / \mathrm{s}$ 'salt' in yellow at depths of about $2100-2250 \mathrm{~m}$, and a thicker, very low velocity $1700 \mathrm{~m} / \mathrm{s}$ region above it in blue at about depth $1750-2100 \mathrm{~m}$.

\section{Residual Processing}

The layer stripping approach described above leads to a propagation of error in both velocity and boundary location which results in a progressive defocusing of the migration with depth. To partially correct for this defocusing, the final depth section is obtained by applying residual moveout correction to the common image-point gathers to flatten each event before stacking.

This residual moveout correction is performed without re-migrating the data. While this does not correct for absolute errors in placement of the reflectors, it does sharpen the stacked image.

Retaining all the image-point gathers also allows us to apply other conventional processing techniques such as mute and filter to improve the appearance of the final migrated section. It is also possible to stack different offset ranges to examine the possibility of 'undershooting.'

Figure 8 shows the result of this residual processing for the Marmousi data. The processing in this case included muting of selected offsets, spectral whitening, and residual moveout correction, primarily in the vicinity of depth $2000 \mathrm{~m}$. The main effect has been to sharpen the reflector that we interpreted as 'top of salt' at about depth $2100 \mathrm{~m}$ and $x$ range $6000-6500 \mathrm{~m}$.

\section{ANALYSIS OF WORKSHOP RESULT}

Our migrated image in figure 8 is well resolved at all depths where our systematic layer stripping velocity analysis was usable. This includes both sides of the model down to around $2000 \mathrm{~m}$, and the faulted central portion to about depth $1200 \mathrm{~m}$. We also imaged some deeper events, such as the steeply-dipping layers at depths $2500-3000 \mathrm{~m}$ and lateral position $4000-4500 \mathrm{~m}$ (on the west side of the pre-salt anticline).

We did not succeed in imaging either the turtleback formation or the pre-salt anticline itself.

The event we called 'top of salt' at depth $2100 \mathrm{~m}$ in the central portion does bear some resemblance to the turtleback structure of the true Marmousi model, although it is too deep and too far east. Actually, the mistakenly slow $1700 \mathrm{~m} / \mathrm{s}$ velocity that appears in our model immediately above this 'salt' event suggests that it is probably an incorrectly positioned image of a considerably deeper event-perhaps a reflector in the pre-salt carbonate section.

\section{POST-WORKSHOP MODEL REFINEMENT}

Careful examination of the amplitude of our depth section indicates that there was a great deal of information in the data that we did not use in generating our model prior to the workshop. Let us consider the events labelled in figure 9: 


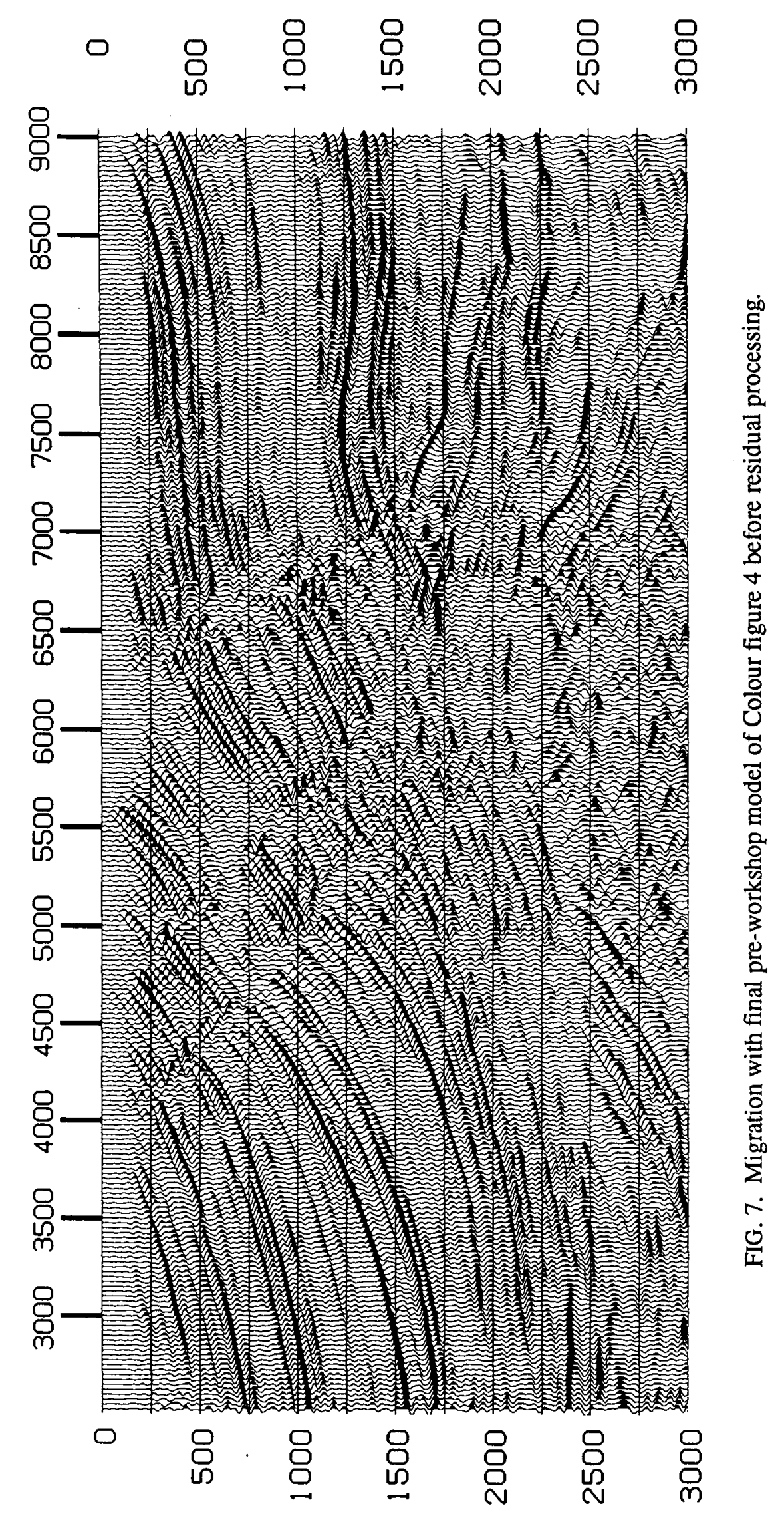




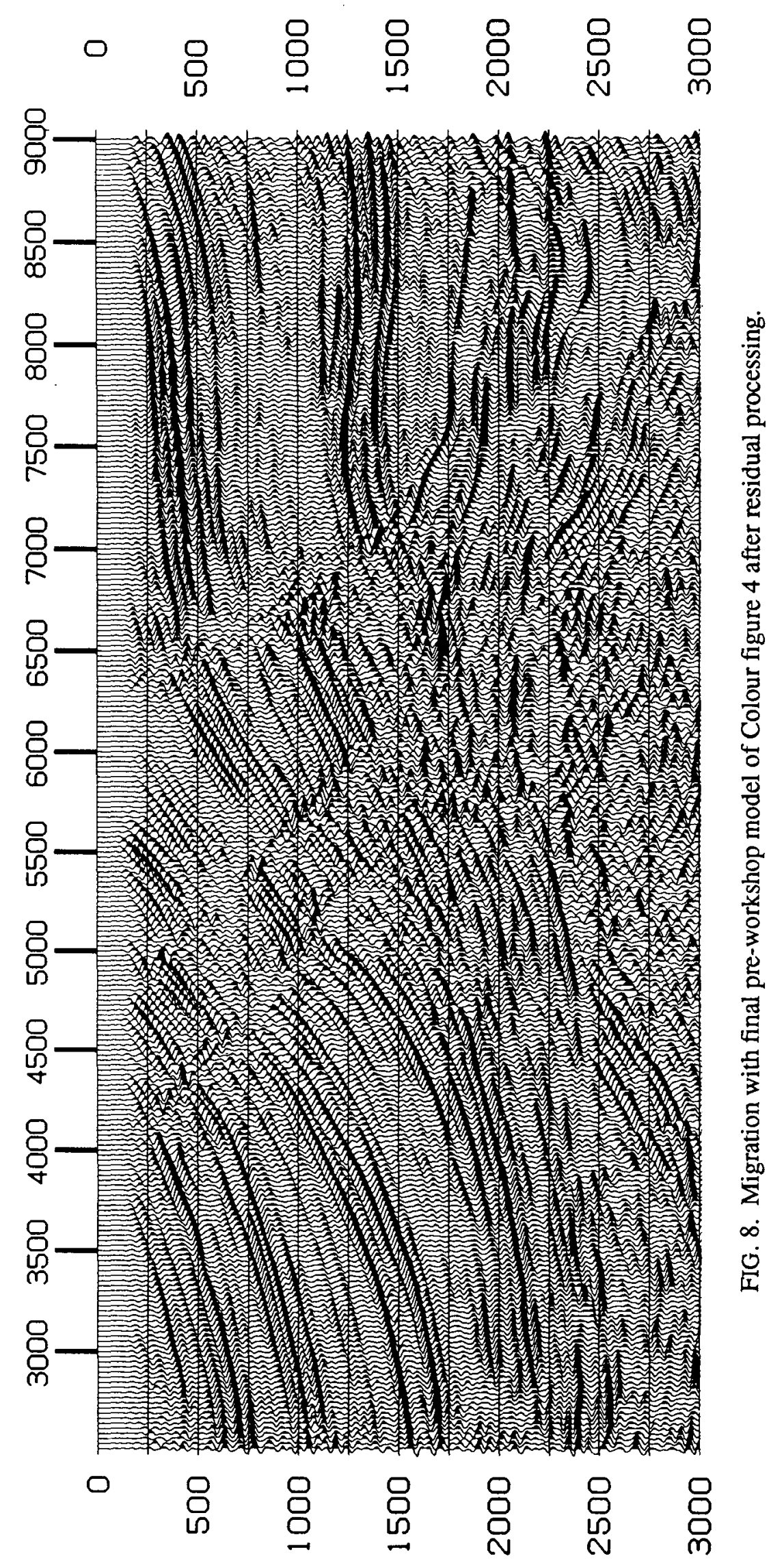


The amplitude of event $A$ changes from very weak $(x=3000 \mathrm{~m})$ to very strong $(x=5000 \mathrm{~m})$, indicating a high velocity layer that is thickening from west to east. (The thinning of layer $D$, on the other hand, is less obvious.) The strong peak B and the strong trough $C$ together hint at the existence of a high velocity layer, truncated by the faults.

The strong troughs at $F$ and $G$ also turn out to be the bases of high velocity chunks of rock. Another high velocity zone, at $\mathbf{H}$, was masked by the errors in our earlier model., but becomes stronger after the shallower high velocity layer bounded by $B$ and $C$ is fitted into the model.

Our original model had compensated for these missing pieces, as well as the misplacement of boundaries $A$ and $D$, by adjusting the velocities of neighboring layers. For example, given our new knowledge that layer $\mathrm{D}$ is thinning out, the region at $x=5000 \mathrm{~m}$, depths $400-900 \mathrm{~m}$, now requires a slightly higher velocity to focus the image at depth $900 \mathrm{~m}$. The errors in our original model, as a result of all the missing pieces, had propagated downward, making the region under $G$ and $H$ nearly impossible to image.

Knowing about these high velocity pieces that were missing from our original model, we re-built the model using the same layer stripping method described earlier, but paying extra attention to the events labelled in Figure 9. Please note that in this process, we did not simply put layer boundaries at the same positions as those of the exact Marmousi model. This was still a 'semi-blind' test, in which we wanted to see how far the data could take us, using just a little extra information that could conceivably have been available from well logs, regional geologic knowledge, etc.

\section{POST-WORKSHOP RESULT}

Our revised model constructed after the workshop is shown in Colour figure 5. The migrated image obtained from this model is in figure 10. No residual processing has been applied to the image in figure 10.

Comparison of figure 10 with our workshop result (figure 8) shows better imaging of the events labelled in figure 9 and of the regions surrounding those events. Our newer results are also more selfconsistent, in the sense that the locations of imaged reflectors better match the boundary locations in the model.

We have still not succeeded in imaging the turtleback formation in the central part of the model. However, our image no longer includes the incorrect 'salt' event which, as noted previously, looked deceptively similar to the desired turtleback structure but was probably a misplaced image of a much deeper reflector.

Perhaps most impressively, the deep (pre-salt) anticline is now visible (except for some dips that are still wrong at about $x=6000 \mathrm{~m}$ ). Note the strong, nearly flat, reflector at depth $2500 \mathrm{~m}$ and $\mathrm{x}$ range $6000-7500 \mathrm{~m}$ in figure 10 . This 'bright spot' appears to match a very low velocity and density layer near the top of the anticline (see Colour figures 2 and 3) which the creators of the Marmousi model apparently intended to simulate a hydrocarbon reservoir.

\section{DISCUSSION}

We have been able to achieve good quality images by constructing migration velocity models with a layer stripping approach using repeated common-offset test migrations. Much of the success of this approach depends simply on having a fast, efficient depth migration program so that many velocities can be tested. For this purpose, the superior speed of Kirchhoff migration makes it the method of choice.

The turtleback formation in the Marmousi model is extremely difficult to image because the high velocity faulted blocks above it act as 'lenses' that severely bend rays passing through them. The data can be correctly migrated only when these high velocity blocks are correctly included in the model. At the same time, these pieces are small and deep enough to make it very difficult for any velocity analysis to handle them.

The most likely reason why we succeeded in migrating some of the events below the turtleback structure was that these events could be imaged by rays that bypassed the troublesome central area where our model was least accurate. 


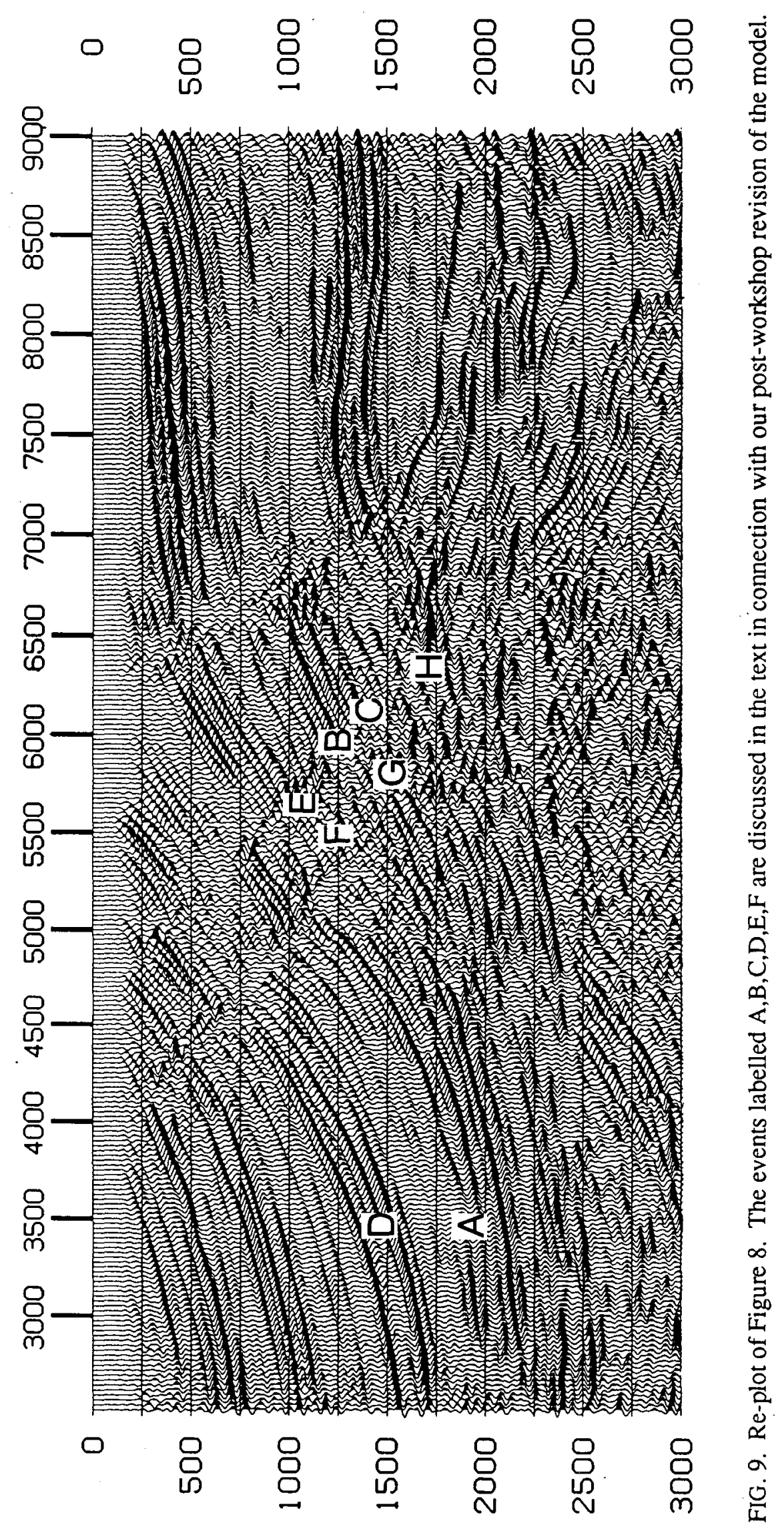




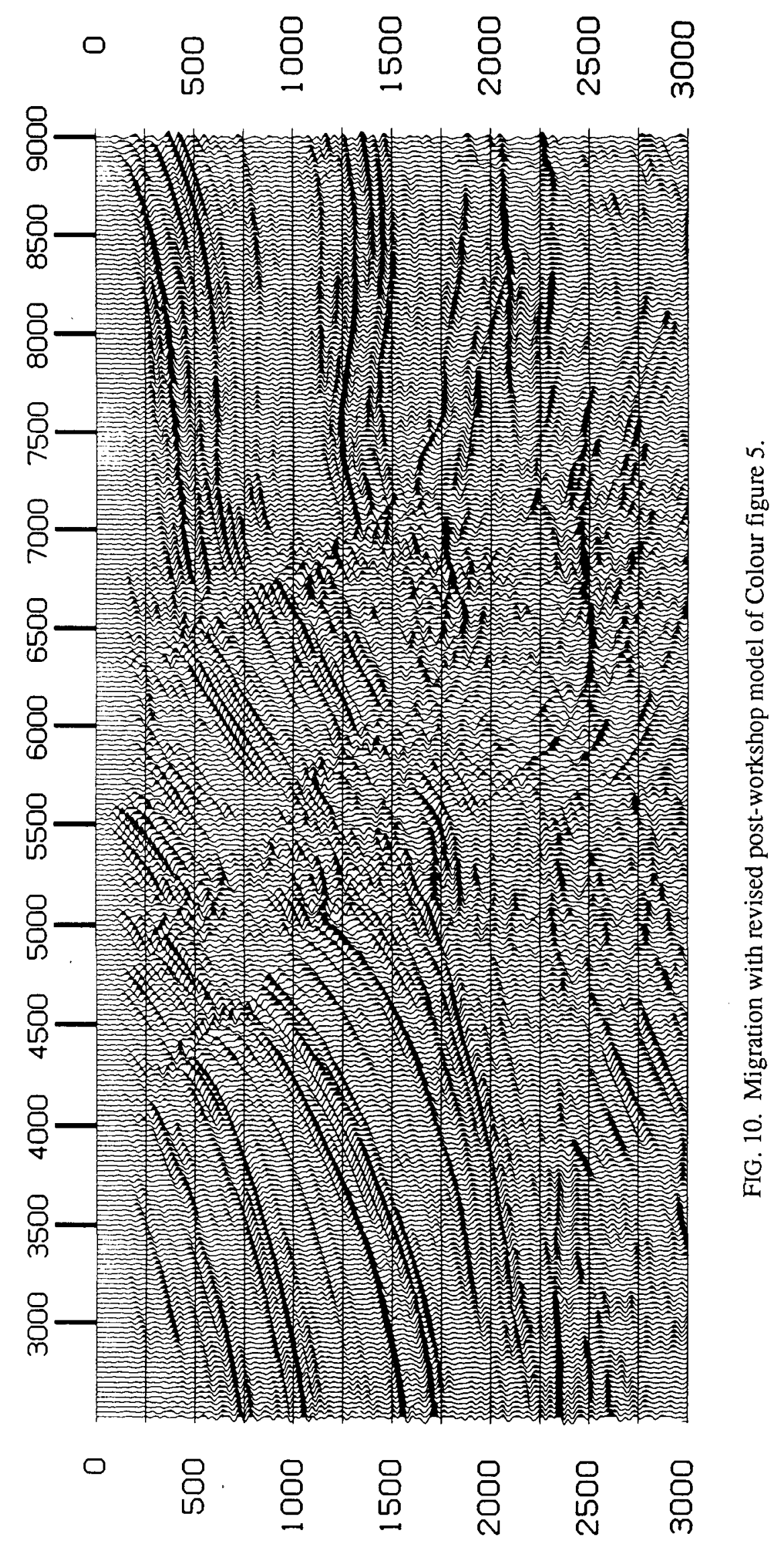


Clearly, the quality of the final migrated image depends on accuracy of the velocity model. It is an open question just how much detail such a model needs to have. For example, a thin (compared with seismic wavelength) layer with essentially parallel edges can probably be omitted without great harm (assuming the average velocity is correct). But larger chunks of material that can act as lenses surely need to be included. Furthermore, where the earth has sharp lateral contrasts in velocity (due to faults or steeply dipping layers), we think these need to be included and must be located very accurately.

We noted that our layer-stripping model building method can break down at sufficiently great depth, in which case we used the 'brute force' method of simply picking the velocity that gives the clearest image. Such an approach is inherently dangerous, and must be well-constrained to give good results. One pitfall inherent in any method relying on focusing of the depth-migrated image is what we call the "lure of the sharper-looking wavelet.' Specifically, wavelets imaged in depth look sharper when migrated with a lower velocity, so one can be tempted to pick too low a velocity.

Our systematic approach based on image-point gathers, when usable, is much more robust. It allows us to judge not only the accuracy, but also the reliability of the picked velocity based on variation of moveout with offset. For example, one could obtain nearly the same stacked image with moveouts that vary monotonically with offset, or sinusoidally with offset. To us, the first case shows a poor choice because the error can be further reduced by a different velocity, but the second case may indicate a good choice because the error cannot be reduced by changing the layer velocity.

When using this or any other layer-stripping method, errors will propagate as the model is extended downward. Consequently, it is important to expend effort at the outset to get the first few layers right. Propagation of errors can also be reduced by constraining the model with external information such as well logs or geologic constraints.

Because our method builds models from the top downward, it is not suitable for refining an already-built model. (The attempt to refine a shal- low layer will lose all deeper information in the model.) However, an already-built model can be refined using travel time tomographic methods (Lines, 1989) or residual moveout velocity analysis methods (van Trier, 1989).

Reflection tomograpy may be considered complementary to our model-building approach. In contrast to our layer-stripping approach which works from the top downward, reflection tomography improves the estimate of shallow velocity structure using information from deep reflections. Thus, a combination of our method with tomographic analysis may result in improved velocity models by working from both top and bottom.

\section{REFERENCES}

FAYE, J.P. and JEANNOT J.P., 1986, Prestack Migration Velocities from Focusing Depth Analysis. Presented at the 56th Annual SEG meeting, Houston.

LINES, L., 1989, Applications of Seismic Traveltime Tomography, a Review. Presented at the SIAM Geophysical Workshop, Houston.

MOSER, T. J., 1989, Efficient Seismic Ray Tracing using Graph Theory. Presented at the 59th Annual SEG meeting, Dallas.

VAN TRIER, J., 1989, Structural Velocity Analysis using Migrated Seismic Data. Presented at the 59th Annual SEG meeting, Dallas.

VAN TRIER, J., and SYMES, W., 1990, Upwind Finite-difference Calculation of Traveltimes. Stanford Exploration Project report 65, 41-58. 